\title{
Minocycline upregulates cyclic AMP response element binding protein and brain-derived neurotrophic factor in the hippocampus of cerebral ischemia rats and improves behavioral deficits
}

This article was published in the following Dove Press journal:

Neuropsychiatric Disease and Treatment

26 February 2015

Number of times this article has been viewed

\section{Yu Zhao' \\ Ming $\mathrm{XiaO}^{2}$ \\ Wenbo $\mathrm{He}^{3}$ \\ Zhiyou $\mathrm{Cai}^{3}$}

'Department of Neurology, the Fourth Affiliated Hospital of Harbin Medical University, Harbin, Heilongjiang Province, People's Republic of China; ${ }^{2}$ Department of Anatomy, Nanjing Medical University, Nanjing, Jiangsu, People's Republic of China; ${ }^{3}$ Department of Neurology, Renmin Hospital, Hubei University of Medicine, Shiyan Renmin Hospital, Shiyan, Hubei Province, People's Republic of China
Correspondence: Zhiyou Cai Department of Neurology, Renmin Hospital, Hubei University of Medicine, Shiyan Renmin Hospital, No 39 Chaoyang Road, Shiyan, Hubei Province, 442000, People's Republic of China

Tel +817198637114

Fax +81 719866 6352

Email c0909@hotmail.com
Background and purpose: The cAMP response element binding protein (CREB) plays an important role in the mechanism of cognitive impairment and is also pivotal in the switch from short-term to long-term memory. Brain-derived neurotrophic factor (BDNF) seems a promising avenue in the treatment of cerebral ischemia injury since this neurotrophin could stimulate structural plasticity and repair cognitive impairment. Several findings have displayed that the dysregulation of the CREB-BDNF cascade has been involved in cognitive impairment. The aim of this study was to investigate the effect of cerebral ischemia on learning and memory as well as on the levels of CREB, phosphorylated CREB (pCREB), and BDNF, and to determine the effect of minocycline on CREB, pCREB, BDNF, and behavioral functional recovery after cerebral ischemia.

Methods: The animal model was established by permanent bilateral occlusion of both common carotid arteries. Behavior was evaluated 5 days before decapitation with Morris water maze and open-field task. Four days after permanent bilateral occlusion of both common carotid arteries, minocycline was administered by douche via the stomach for 4 weeks. CREB and pCREB were examined by Western blotting, reverse transcription polymerase chain reaction, and immunohistochemistry. BDNF was measured by immunohistochemistry and Western blotting.

Results: The model rats after minocycline treatment swam shorter distances than control rats before finding the platform $(P=0.0007)$. The number of times the platform position was crossed for sham-operation rats was more than that of the model groups in the corresponding platform location $(P=0.0021)$. The number of times the platform position was crossed for minocycline treatment animals was significantly increased compared to the model groups in the corresponding platform position $(P=0.0016)$. CREB, pCREB, and BDNF were downregulated after permanent bilateral occlusion of both common carotid arteries in the model group. Minocycline increased the expression of CREB, pCREB, and BDNF, and improved cognitive suffered from impairment of permanent bilateral occlusion of both common carotid arteries.

Conclusion: Minocycline improved cognitive impairment from cerebral ischemia via enhancing CREB, pCREB, and BDNF activity in the hippocampus.

Keywords: vascular cognitive impairment, cAMP response element binding protein, cerebral ischamia, neuroprotection

\section{Introduction}

Increasing findings have evidenced that cerebral ischemia plays a critical role in the pathogenesis of vascular cognitive impairment, and the reduction of cerebral blood flow 
correlates with the severity of cognitive impairment. ${ }^{1,2}$ Various mechanisms of neuronal injury suffered from cerebral ischemia have been proposed, including formation of free radicals, oxidative stress, ${ }^{3,4}$ mitochondrial dysfunction,, 5 inflammatory processes, ${ }^{7}$ genetic factors, environmental impact factors, ${ }^{8,9}$ apoptosis, ${ }^{10}$ and so on. These factors may interact with and amplify each other in a vicious cycle of toxicity, leading to neuronal dysfunction and cognitive impairment. The transcription factor cyclic AMP response element binding protein (CREB) and neurotrophin brain-derived neurotrophic factor (BDNF) have emerged as molecules that may play an important role in modulating mood, behavior, and memory. ${ }^{11-13}$ CREB and BDNF are known to be dysregulated in animal models and in patients suffering from cerebral ischemia, and are deemed to be therapeutic targets of cerebral ischemia. ${ }^{14,15}$

Minocycline, a tetracycline derivative, protects against cerebral ischemia via inhibiting inflammation, oxidative stress, and apoptosis. ${ }^{16,17}$ Previously, we have found that minocycline retarded astrocytic reactivation, and restrained oxidative stress and neuroinflammation in the hippocampus of cerebral ischemia rats. ${ }^{18,19}$ In the present study, we observed the expression of CREB, phosphorylated CREB (pCREB), and BDNF in the hippocampus of cerebral ischemia rats with cognitive impairment by permanent bilateral occlusion of both common carotid arteries, and explored the neuroprotective mechanism of minocycline for the treatment of cerebral ischemia injury. We found that CREB, pCREB, and BDNF were downregulated after permanent bilateral occlusion of both common carotid arteries in a model group, and minocycline attenuated cognitive impairment and upregulated CREB, pCREB, and BDNF in the hippocampus of rats with permanent bilateral occlusion of both common carotid arteries. Therefore, a hypothesis was made that minocycline upregulated CREB, pCREB, and BDNF and improved cognitive impairment from cerebral vascular factors.

\section{Materials and methods Animal and drug}

Wistar rats (10 weeks old, female, quality 200-250 g, from the Field Zoology Research Institute of the Third Military Medical University of the People's Republic of China) were randomly divided into sham-operated group (S) (with a mean survival time of 16 weeks), ischemia model group (M) (with permanent bilateral occlusion of both common carotid arteries), and minocycline treatment group (MT) (beginning treatment after 4 days from permanent bilateral occlusion of both common carotid arteries, minocycline was administered by douche via the stomach for 4 weeks). M and MT groups were separately subdivided into 4-, 8-, and 16-week groups. Each group had six animals. The animal model of cerebral ischemia was established with permanent bilateral occlusion of both common carotid arteries for chronic bilateral common carotid artery occlusion (bCCAo) ${ }^{20,21}$

Rats were anesthetized with $10 \%$ chloral hydrate $(350 \mathrm{mg} / \mathrm{kg}$, intraperitoneally) and breathed normally throughout the surgical procedure. Both common carotid arteries were exposed via a midline cervical incision and doubly-ligated with silk suture. Sham-operated animals were treated in the same manner, except that the common arteries were not ligated. The investigation was performed according to the Guide for the Care and Use of Laboratory Animals published by the US National Institutes of Health. ${ }^{22}$ The animal experiments were performed according to internationally followed ethical standards and approved by the research ethics committee of Chongqing Medical University, Chongqing, People's Republic of China.

Minocycline (100 mg/capsule; Huishi Pharmaceutical Limited Company, Shanghai, the People's Republic of China) was diluted to $0.5 \mathrm{mg} / \mathrm{mL}$ density by normal saline. $\mathrm{S}$ and $\mathrm{M}$ groups were given the same volume of normal saline through douche via the stomach. MT group was given $50 \mathrm{mg} / \mathrm{kg} / \mathrm{d}$ minocycline through douche via the stomach. The minocycline dosage used for animals was as described elsewhere. ${ }^{16,17}$

\section{Morris water maze task}

The Morris water maze task (Chinese Academy of Medical Sciences, People's Republic of China) includes a place navigation test and spatial probe test, and is widely used in behavioral neuroscience to study spatial learning and memory. ${ }^{23}$ The rats were placed in a large circular pool with an invisible platform that allows them to escape the water. The time it took a rat to find the platform and escape was measured for up to four trials a day for 5 days. The time it took to find the platform is referred to as escape latency (the earliest learning measure). After training was complete, the spatial probe trial was conducted in which the escape platform was removed from the pool and the animal allowed to swim for 120 seconds. The spatial probe test was for the measurement of preservation-of-experience (memory) capacity, ie, looking for the platform position. The time it took to find the unmoved platform (learning latency) and the times a rat crossed the corresponding position of the removed platform in 120 seconds (memory latency) were recorded.

\section{Immunohistochemical assay}

Tissue samples were collected after surgery and immediately frozen with liquid nitrogen. Prior to immunohistochemistry 
assay, frozen sections were prepared with a cryostat (FACS caliber; Becton Dickinson, Franklin Lakes, NJ, USA) at $-20^{\circ} \mathrm{C}$, dried at room temperature, and fixed with acetone. The peripheral blood mononuclear cells were routinely isolated and the slides were prepared with a cytospin. The avidin-biotin-peroxidase complex immunohistochemical assay was carried out according to the protocols we described before anti-CREB (Sigma-Aldrich Co., St Louis, MO, USA), anti-pCREB (Sigma-Aldrich Co.), and anti-BDNF (Santa Cruz, LA, CA, USA) were prepared. The second antibody, a goat anti-mouse IgG labeled with biotin, was purchased from Vector Co. (Burlingame, CA, USA). Two hundred cells were counted and the intensity of staining for each of those cells was adjusted. Five grades were employed to express the degrees of staining, which represent five reaction coefficients, respectively. The five products of every coefficient and the corresponding cell number were added up, which resulted in the value of a positive score. ${ }^{16,18}$ All slides were measured in duplicate. Those samples with a positive score over 10 or a frequency over 5\% were considered as positive.

\section{Western blotting}

Rat tissues were dissected and homogenized in Tissue Protein Extraction Reagent (T-PER) buffer in the presence of protease inhibitors. After homogenization, the lysates were centrifuged at $100,000 \times g$, and the supernatants were saved for Western blotting. Equal amounts of lysates were subject to sodium dodecyl sulfate-polyacrylamide gel electrophoresis and Western blotting analysis using antibodies specific for the following: CREB (1:1,000; BioSource International,
Inc., USA), pCREB (1:1,000; BioSource International, Inc.), BDNF (1:500; Sigma-Aldrich Co., St Louis, MO, USA), and $\beta$-tublin (1:200; BioSource International Inc., Camarillo, CA, USA). The optical densities of the specific bands were scanned and measured by image analysis software (Tongji Qianping Company, Wuhan, Hubei Province, People's Republic of China).

\section{Statistical analysis}

Quantitative data were expressed as mean \pm standard deviation. All statistical analyses used the SPSS software for Windows 13.0 (SPSS, Inc., Chicago, IL, USA) and Student's $t$-test for intergroup analysis. Student-Newman-Keuls test was performed when variance was equal, and Games-Howell test was performed when variance was not equal. Pearson's correlation analysis was also performed on some indices. $P<0.05$ was considered as statistically significant.

\section{Results}

\section{Minocycline improved behavioral deficits}

Cerebral ischemia was induced in 10-week-old Wistar rats by bCCAo as described previously. ${ }^{18,19}$ After the performance of bCCAo, rats were subjected to the Morris water maze. Escape latency decreased after 1 day of training. On days 3, 4, and 5, $\mathrm{S}$ animals immediately swam toward the platforms in the water maze, whereas $M$ rats swam longer distances before finding the platform (Figure 1A). In general, escape latency decreased with bCCAo duration $(P=0.0004)$, the $\mathrm{M}$ rats, after minocycline treatment, swam shorter distances than control rats before finding the platform $(P=0.0007)$ (Figure 1A).
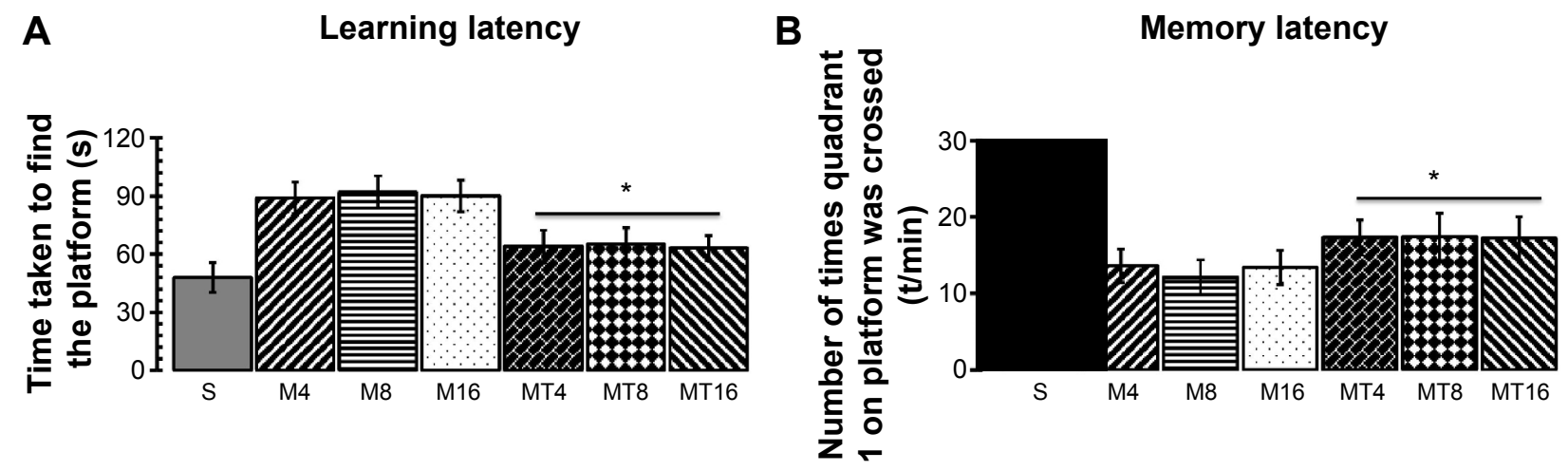

Figure I Morris water maze performance.

Notes: Rats were randomly divided into S (with a mean survival time of 16 weeks), $M$ (with permanent bilateral occlusion of both common carotid arteries), and MT (after 4 days of permanent bilateral occlusion of both common carotid arteries, minocycline was administered by douche via the stomach for 4 weeks) groups. The $M$ and MT groups were subdivided further into 4-, 8-, and I6-weeks groups, with six rats in each group. (A) During place navigation (learning), S rats swam shorter distances than $M$ animals before finding the platform $(* P<0.01)$ and escape latency gradually decreased with the duration of cerebral ischemia after $M T$ ( $* P<0.0 \mathrm{I})$. (B) In the probe trials (memory), the number of times the platform position was crossed for $\mathrm{S}$ rats was more than for the bCCAo rat groups in the corresponding platform location $(* P<0.0 \mathrm{I})$. The number of times the platform position was crossed for MT animals was significantly increased compared to the bCCAo rat groups in the corresponding platform position $(* P<0.01)$.

Abbreviations: bCCAo, bilateral common carotid artery occlusion; M, ischemia model; M4, bCCAo 4 weeks; M8, bCCAo 8 weeks; MI6, bCCAo I6 weeks; MT, minocycline treatment; MT4, MT 4 weeks; MT8, MT 8 weeks; MTI6, MT 16 weeks; S, sham-operated. 
In the probe trials, the number of times the platform position was crossed for the $\mathrm{S}$ group was more than for the bCCAo rats groups in the corresponding platform location $(P=0.0000)$ (Figure 1B). The number of times the platform position was crossed for MT animals was significantly increased compared to bCCAo rat groups in the corresponding platform position $(P=0.0016)$ (Figure 1B).

\section{Minocycline upregulated CREB and $P C R E B$}

The results of immunohistochemistry and Western blotting showed that expression of CREB and pCREB in the MT group was significantly higher than that of the $M$ group at the corresponding time. Expression of CREB and pCREB by immunohistochemistry in the $\mathrm{M}$ groups was more decreased than in the control group ( $P=0.0009 ; P=0.0023)$, whereas expression of CREB and $\mathrm{pCREB}$ in the MT groups was more increased than in the control groups $(P=0.0001 ; P=0.0005)$ (Figure 2). Expression of CREB and pCREB by Western blotting in the $\mathrm{M}$ groups was lowered more than in the control group ( $P=0.0010 ; P=0.0031)$, whereas expression of CREB and pCREB in the MT groups was more enhanced than in the control groups $(P=0.0004 ; P=0.0003)$ (Figure 2$)$.

\section{Minocycline enhanced BDNF activity}

The results of immunohistochemistry showed that expression of BDNF in the MT animals was higher than that of the $\mathrm{M}$ ones $(P=0.0005)$, whereas expression of BDNF in the $\mathrm{M}$ groups was decreased compared to the control group $(P=0.0001)$. Western blotting analysis found that BDNF in the MT groups was higher than that of the $M$ group $(P=0.0006)$, while expression of BDNF in the $\mathrm{M}$ groups was more reduced than in the control group $(P=0.0000)$ (Figure 3$)$.

\section{Correlation between cognition and CREB, $P C R E B$, and BDNF}

The number of times the platform position was crossed during the probe trial for the $\mathrm{S}$ group was higher than for $\mathrm{M}$ rats $(P=0.0021)$. Therefore, we determined correlation analysis between the number of times the platform position was crossed and CREB, pCREB, and BDNF expression. Linear correlation analysis shows that the optical density (OD) values of immunoblotting protein for CREB, pCREB, and BDNF were negatively correlated with the number of platform position crossings $(r=-0.314, P=0.0062$; $r=-0.352, P=0.0004 ; r=-0.381, P=0.0031)$ respectively (Figure 4). We inferred that downregulation of CREB,

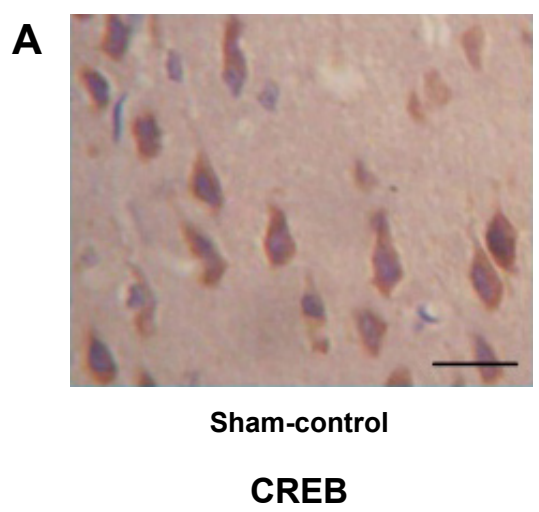

CREB

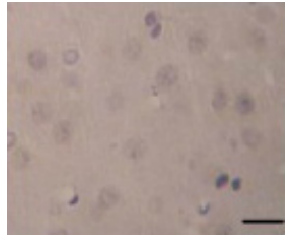

M4

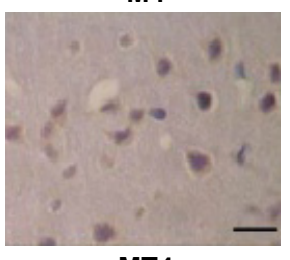

MT4

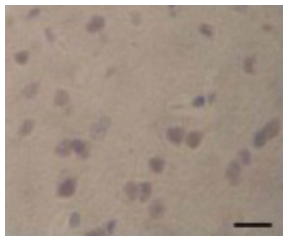

M8

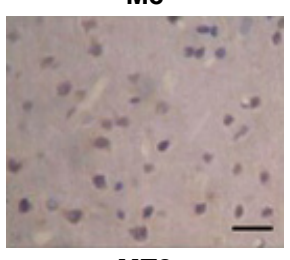

MT8

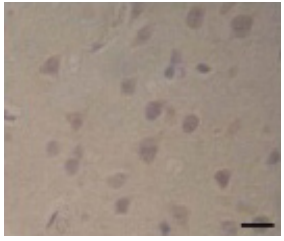

M16

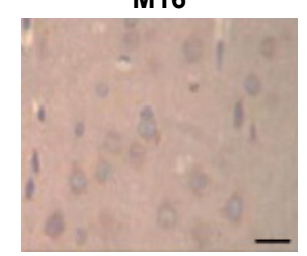

MT16

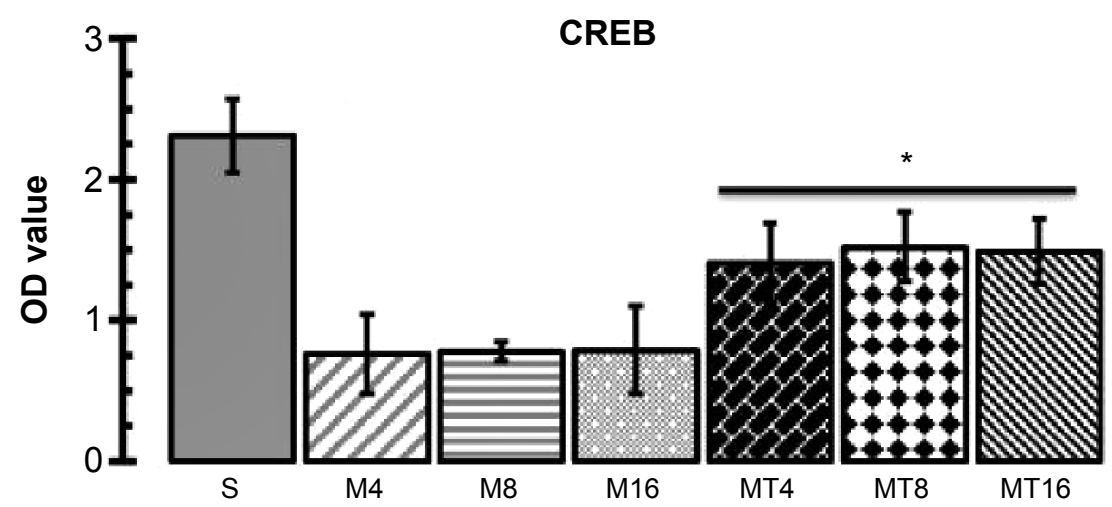

Figure 2 (Continued) 


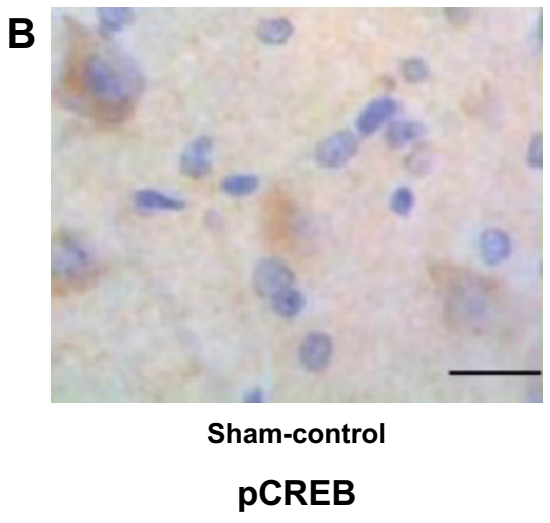

PCREB

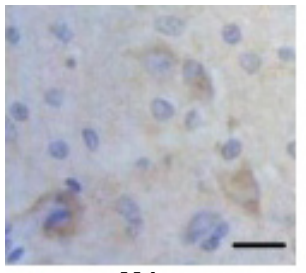

M4

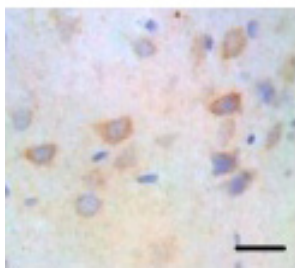

MT4

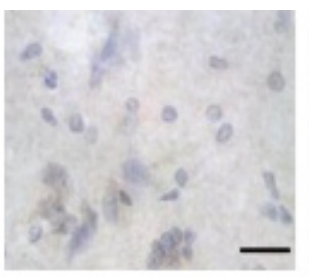

M8

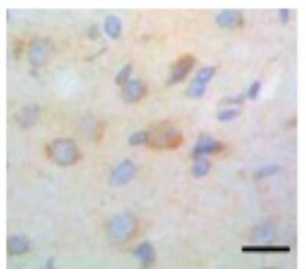

MT8

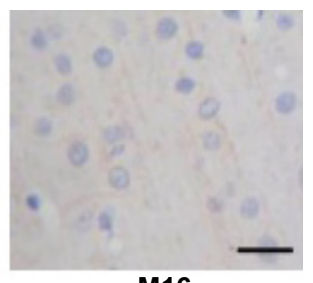

M16

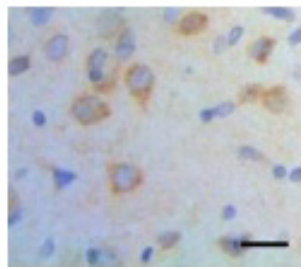

MT16

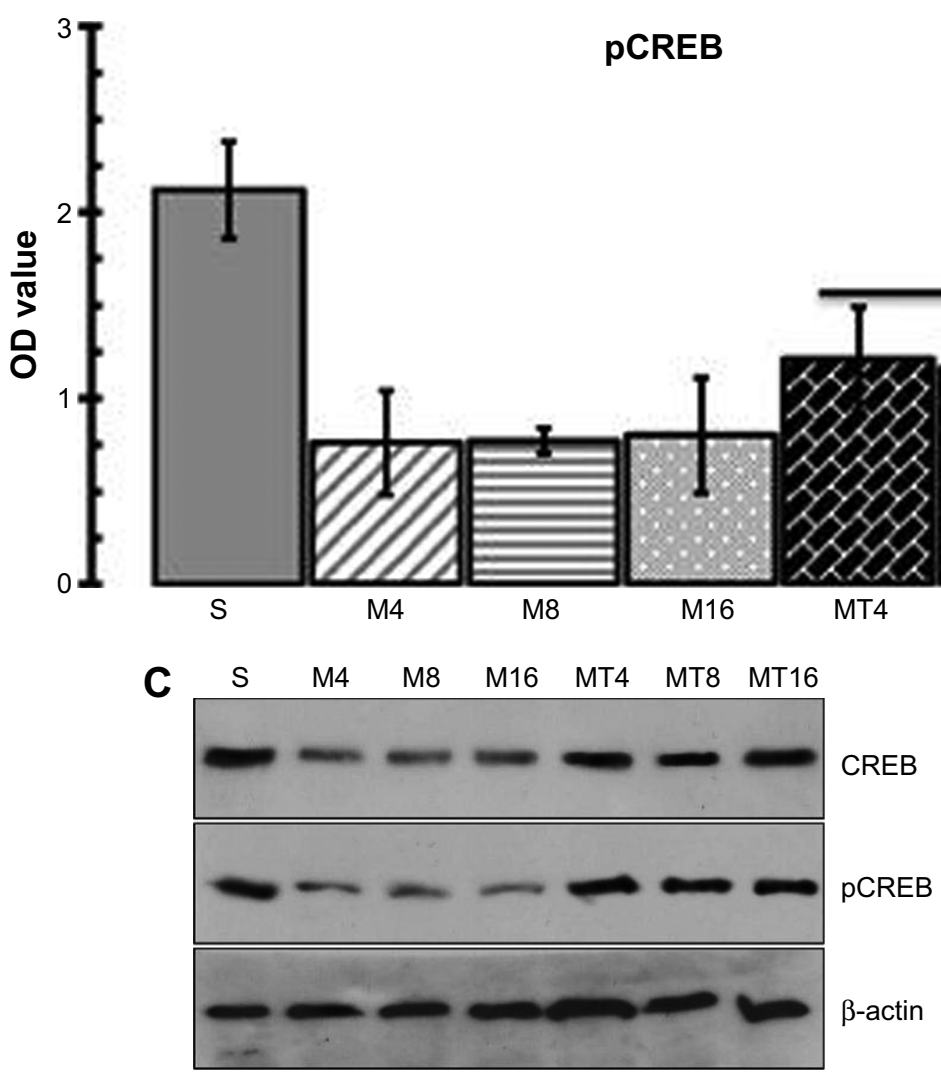

Figure 2 The expression of CREB and PCREB in the hippocampus by immunohistochemistry and Western blotting.

Notes: (A, B) The expression of CREB and PCREB in the MT groups was higher than that of the M groups $(* P<0.0 \mathrm{I})$. The scale bar is $25 \mu \mathrm{m}$. (C) Western blotting revealed that the MT groups had significantly higher levels of CREB and $P C R E B$ than in the $M$ groups $(* P<0.0 I)$.

Abbreviations: bCCAo, bilateral common carotid artery occlusion; CREB, cAMP response element binding protein; M4, bCCAo 4 weeks; M8, bCCAo 8 weeks; MI6, bCCAo 16 weeks; MT4, minocycline treated 4 weeks; MT8, minocycline treated 8 weeks; MTI6, minocycline treated I6 weeks; OD, optical density; pCREB, phosphorylated CREB; S, sham-operation group.

pCREB, and BDNF contributed to cognitive impairment from chronic cerebral ischemia.

\section{Discussion}

Minocycline, a semisynthetic tetracycline antibiotic that effectively crosses the blood-brain barrier, has been reported to have significant neuroprotective effects in cognitive impairment, ${ }^{24,25}$ schizophrenia, ${ }^{26}$ cerebral ischemia, ${ }^{27}$ amyotrophic lateral sclerosis, ${ }^{28}$ Alzheimer's disease, ${ }^{25,29}$ Huntington's disease, ${ }^{30,31}$ and Parkinson's diseases. ${ }^{32}$ Minocycline can inhibit ischemic-induced inflammation, ${ }^{33,34}$ astrocyte reactivation, ${ }^{35}$ microglia activation, ${ }^{32}$ oxidative stress, ${ }^{36,37}$ apoptosis, ${ }^{37,38}$ and so on. One common manifestation after brain ischemic damage is cognitive impairment. In this 
A

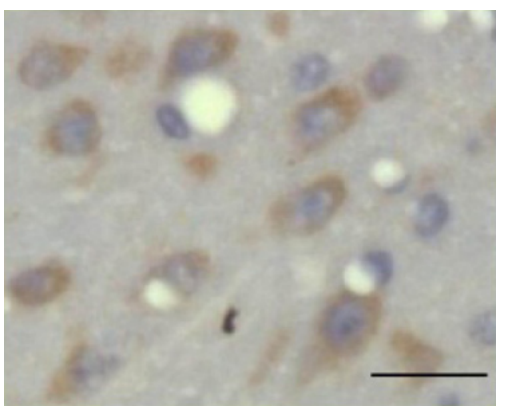

Sham-control
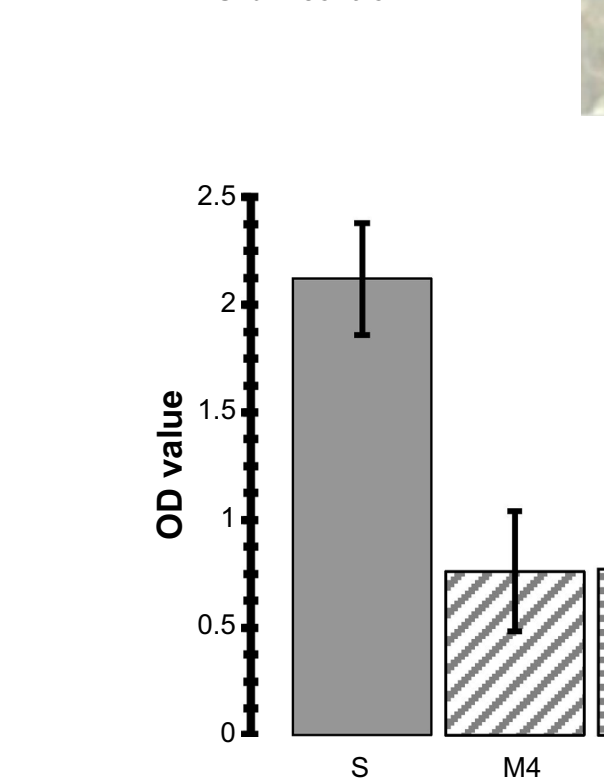

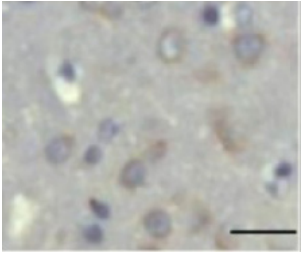

M4

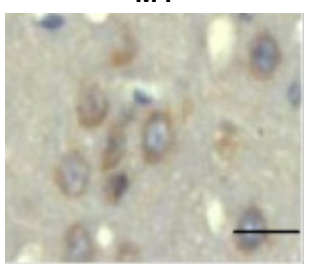

MT4

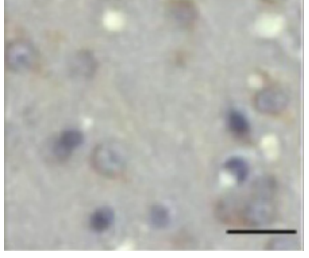

M8

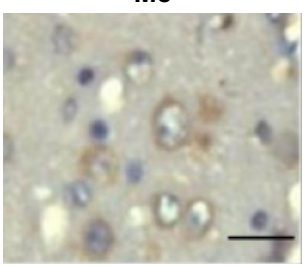

MT8

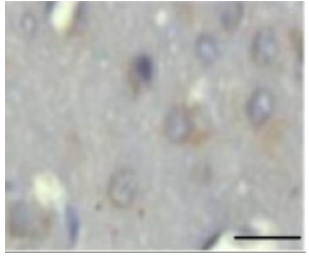

M16

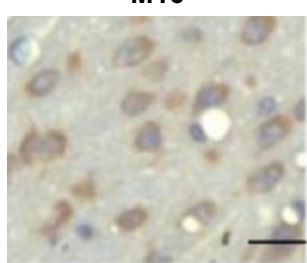

MT16

Immunohistochemistry

B
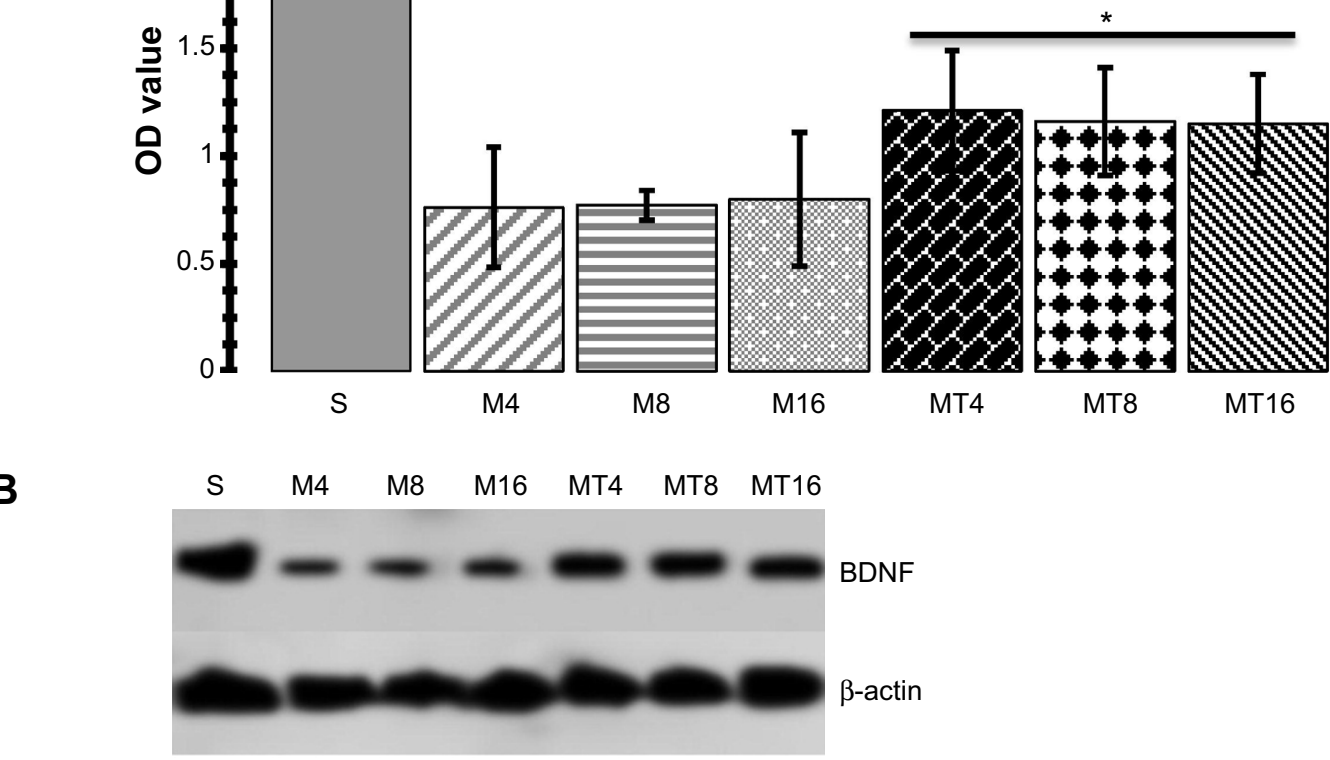

MT4

MT8

MT16

Figure 3 Assessment of BDNF.

Notes: $(\mathbf{A})$ Assay of protein level of BDNF by immunohistochemistry. Optical density value in $M T$ and $S$ groups was significantly higher than that of $M$ groups $(P<0.01)$. Expression of BDNF in MT was more increased compared to control group (M) $(P<0.00 \mathrm{I})$. The scale bar is $25 \mu \mathrm{m}$. (B) assay of BDNF by immunoblotting. The level of BDNF in control groups $(M)$ was more decreased than in the MT groups $(P<0.00 \mathrm{I})$. Expression of BDNF in the MT and $S$ groups was more increased than in the control groups $(M)(P<0.001)$.

Abbreviations: bCCAo, bilateral common carotid artery occlusion; BDNF, brain-derived neurotrophic factor; M, ischemia model; M4, bCCAo 4 weeks; M8, bCCAo 8 weeks; MI6, bCCAo 16 weeks; MT, minocycline treatment; MT4, MT 4 weeks; MT8, MT 8 weeks; MTI6, MT I6 weeks; OD, optical density; S, sham-operation.

present study, we established the cerebral ischemia model by a permanent bilateral occlusion of both common carotid arteries. The results from Morris water maze test showed that cognitive impairment occurred with the ischemic brain damage model, and cognitive impairment of control animals had been attenuated after minocycline administration. Furthermore, minocycline increased the levels of CREB, pCREB, and BDNF in the hippocampus of rats by a permanent bilateral occlusion of both common carotid arteries.
The results from the Morris water maze test showed that cognitive impairment occurred with chronic cerebral ischemia injury, and minocycline reduced cognitive impairment caused by permanent bilateral occlusion of both common carotid arteries. To further examine the mechanism by which cognitive impairment occurred with chronic cerebral ischemia and by which minocycline improved behavioral deficits, the expression of CREB, a bio-marker of memory, ${ }^{39}$ was examined in the hippocampus tissue of rats. 

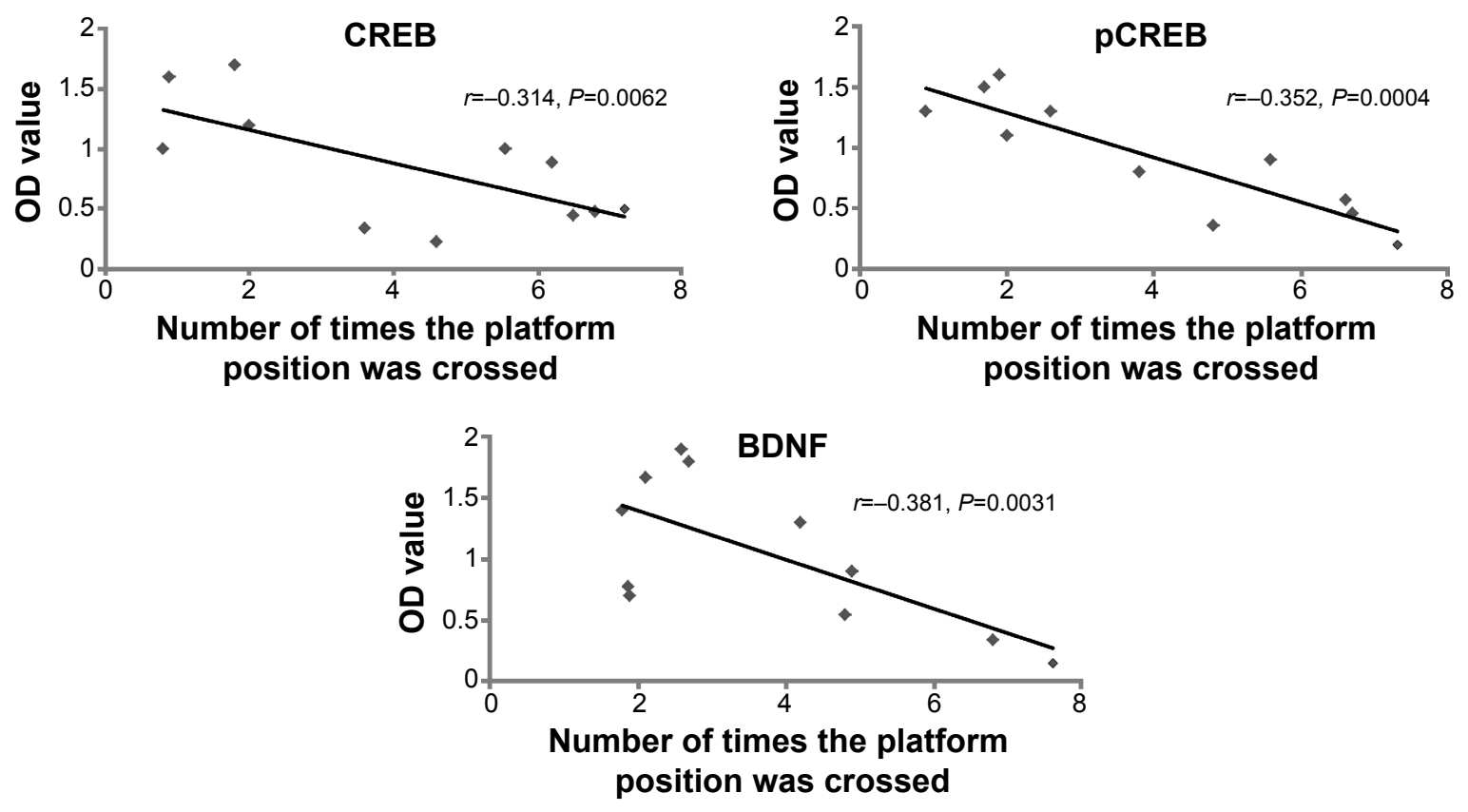

Figure 4 Correlation between cognition and CREB, PCREB, and BDNF.

Notes: Correlation between the number of times that the platform position was crossed and OD value of immunoblotting protein for CREB, $\mathrm{PCREB}$, and BDNF. Abbreviations: BDNF, brain-derived neurotrophic factor; CREB, cAMP response element binding protein; OD, optical density; $P C R E B$, phosphorylated CREB.

CREB, belonging to the family of leucine zipper transcription factors, is critical to induce its effects at phosphorylation of a serine residue (S133) in its kinase-inducible domain. Phosphorylation of CREB can be accomplished by a number of upstream signaling cascades. ${ }^{40,41}$ Studies indicate that these pathways are perturbed in patients suffering from cognitive impairment and they are also known to be influenced by anti-cognitive impairment treatment. ${ }^{42,43} \mathrm{CREB}$ has a role to play in the pathogenesis of cognitive impairment and in anti-cognitive impairment action. ${ }^{39,44,45}$ In the present study, both CREB and pCREB were downregulated after cerebral ischemia injury, and downregulation of CREB and pCREB contributed to cognitive impairment from cerebral ischemia injury by correlation analysis. Both CREB and PCREB were upregulated in the hippocampus tissue after minocycline administration. In addition, the cAMP-CREB signaling cascade is critical to the generation of new neurons in the rodent hippocampus, and also facilitates their subsequent morphological maturation. Thus, CREB's neuroprotective and survival-enhancing properties can act in a manner analogous to that of anti-cognitive impairment. Therefore, minocycline can mediate overexpression of CREB in the hippocampus and has an anti-cognitive impairment-like effect in the process of cerebral ischemia injury.

To further clarify the mechanism by which minocycline improved behavioral deficits, the expression of BDNF (a neurotrophin that play a critical role in the development of the brain and continues to have a seminal role in shaping plasticity in the mature nervous system) was investigated. BDNF is the most widely expressed member of the nerve growth factor family of growth regulators, collectively termed the neurotrophins. ${ }^{46-48}$ The neurotrophins play a critical role in the development of the brain and continue to have a seminal role in shaping plasticity in the mature nervous system. ${ }^{49} \mathrm{BDNF}$ has also been shown to elicit rapid action potentials, thus influencing neuronal excitability, and it has a demonstrable role in activity-dependent synaptic plasticity events like longterm potentiation, learning tasks, and memory. ${ }^{50,51} \mathrm{BDNF}$ is involved in structural remodeling, neuronal plasticity, and synaptic restructuring, ${ }^{52,53}$ and is promising as a candidate molecule underlying the structural changes associated with cerebral ischemia damage, and as a potential target for cerebral ischemia damage. ${ }^{54}$ In the present study, BDNF was downregulated in the hippocampus tissue after chronic cerebral ischemia injury, whereas BDNF was upregulated after minocycline administration. Thus, it is speculated that minocycline has an anti-cognitive impairment-like effect in behavioral models of vascular cognitive impairment through enhancing the expression of BDNF in the hippocampus.

The hippocampus is a key limbic region whose structure and function is compromised in cognition disorders. In the hippocampus, increased activity of the CREB-BDNF cascade results in anti-cognition responses. ${ }^{13,55,56}$ Hippocampal overexpression of BDNF and CREB is capable of mimicking 
both the structural consequences of sustained anti-cognition treatment as well as exerting anti-cognition-like behavioral effects. ${ }^{56}$ Activation of the CAMP-CREB cascade results in increased neurogenesis of dentate granule cell progenitors, and increased dendritic length and branching. It is possible that CREB, a transcriptional activator of BDNF, recruits this neurotrophin to mediate its effects on structural plasticity. ${ }^{57,58}$ BDNF, in addition to being a target of CREB, can itself recruit this particular transcription factor by activating the MAP kinase cascade, thus setting up a potential positive feedback loop. Taken together, elevated CREB-BDNF, through its protective influences on vulnerable hippocampal neurons and ability to directly promote structural reorganization, could result in repair of the region known to be damaged in cognitive impairment. Moreover, the well-established role of BDNF and CREB in hippocampal-dependent learning and memory may play a critical role in ameliorating the cognitive symptoms. ${ }^{13,59}$ In the present study, minocycline efficiently improved behavioral deficits and increased CREB, pCREB, and BDNF that had been down-regulated by cerebral ischemia. It is possible that minocycline recruits CREB-BDNF cascade to mediate its effects on structural plasticity and set up a potential positive CREB-BDNF feedback loop. Taken together, elevated CREB-BDNF activity by minocycline, through its protective influences on vulnerable hippocampal neurons and ability to directly promote structural reorganization, could result in repair of the region known to be damaged in cognitive impairment.

\section{Conclusion}

In conclusion, this study is the first to evaluate the influence of minocycline on the transcription factors (CREB, pCREB, and BDNF) as potential key players in the treatment of vascular cognitive impairment in the process of cerebral ischemia injury. From a clinical point of view, the ability of minocycline to modulate cognitive impairment may be of great importance in the selection of neuroprotective agents, especially in chronic cerebral ischemia procedures.

\section{Acknowledgments}

This work was supported by the Youth Provincial Nature Science Foundation of Heilongjiang grant (QC2011C053) to Dr Yu Zhao, the Provincial Nature Science Foundation of Anhui grant (1308085MH158) to Dr Zhiyou Cai, and funding for basic research from the Ministry of Civil Affairs (Number: 2007-18-3-05) to Dr Zhiyou Cai.

\section{Disclosure}

The authors report no conflicts of interest in this work.

\section{References}

1. Briones TL, Woods J, Wadowska M. Chronic neuroinflammation and cognitive impairment following transient global cerebral ischemia: role of fractalkine/CX3CR1 signaling. J Neuroinflammation. 2014;11:13.

2. Lei Y, Guo Q, Li Y, Jiang H, Ni W, Gu Y. [Characteristics of cognitive impairment in adults with cerebral ischemia]. Zhonghua Yi Xue Za Zhi. 2014;94(13):984-989. Chinese.

3. Ge P, Zhao J, Li S, Ding Y, Yang F, Luo Y. Inhalation of hydrogen gas attenuates cognitive impairment in transient cerebral ischemia via inhibition of oxidative stress. Neurol Res. 2012;34(2):187-194.

4. Raz L, Zhang QG, Zhou CF, et al. Role of Rac1 GTPase in NADPH oxidase activation and cognitive impairment following cerebral ischemia in the rat. PLoS One. 2010;5(9):e12606.

5. Li J, Ma X, Yu W, et al. Reperfusion promotes mitochondrial dysfunction following focal cerebral ischemia in rats. PLoS One. 2012;7(9): e46498.

6. Li J, Yu W, Li XT, Qi SH, Li B. The effects of propofol on mitochondrial dysfunction following focal cerebral ischemia-reperfusion in rats. Neuropharmacology. 2014;77:358-368.

7. Feng X, Yang S, Liu J, et al. Electroacupuncture ameliorates cognitive impairment through inhibition of NF- $\mathrm{KB}$-mediated neuronal cell apoptosis in cerebral ischemia-reperfusion injured rats. Mol Med Rep. 2013; 7(5):1516-1522.

8. Winocur G, Thompson C, Hakim A, Greenwood C. The effects of high- and low-risk environments on cognitive function in rats following 2-vessel occlusion of the carotid arteries: a behavioral study. Behav Brain Res. 2013;252:144-156.

9. Gobbo OL, O'Mara SM. Impact of enriched-environment housing on brain-derived neurotrophic factor and on cognitive performance after a transient global ischemia. Behav Brain Res. 2004;152(2):231-241.

10. Cheng Z, Li L, Mo X, et al. Non-invasive remote limb ischemic postconditioning protects rats against focal cerebral ischemia by upregulating STAT3 and reducing apoptosis. Int J Mol Med. 2014;34(4):957-966.

11. Yu S, Cheng Q, Li L, Liu M, Yang Y, Ding F. 2-(4-Methoxyphenyl) ethyl-2-acetamido-2-deoxy- $\beta$-d-pyranoside confers neuroprotection in cell and animal models of ischemic stroke through calpain1/PKA/ CREB-mediated induction of neuronal glucose transporter 3. Toxicol Appl Pharmacol. 2014;277(3):259-269.

12. Chung JY, Kim MW, Bang MS, Kim M. Increased expression of neurotrophin 4 following focal cerebral ischemia in adult rat brain with treadmill exercise. PLoS One. 2013;8(3):e52461.

13. Gumuslu E, Mutlu O, Sunnetci D, et al. The Antidepressant Agomelatine Improves Memory Deterioration and Upregulates CREB and BDNF Gene Expression Levels in Unpredictable Chronic Mild Stress (UCMS)Exposed Mice. Drug Target Insights. 2014;8:11-21.

14. Yao C,Zhang J,Liu G, Chen F, Lin Y. Neuroprotection by (-)-epigallocatechin3 -gallate in a rat model of stroke is mediated through inhibition of endoplasmic reticulum stress. Mol Med Rep. 2014;9(1):69-76.

15. Qin L, Jing D, Parauda S, et al. An adaptive role for BDNF Val66Met polymorphism in motor recovery in chronic stroke. J Neurosci. 2014; 34(7):2493-2502.

16. Cai Z, Yan Y, Wang Y. Minocycline alleviates beta-amyloid protein and tau pathology via restraining neuroinflammation induced by diabetic metabolic disorder. Clin Interv Aging. 2013;8:1089-1095.

17. Cai Z, Zhao Y, Yao S, Bin Zhao B. Increases in $\beta$-amyloid protein in the hippocampus caused by diabetic metabolic disorder are blocked by minocycline through inhibition of NF- $\mathrm{\kappa B}$ pathway activation. Pharmacol Rep. 2011;63(2):381-391.

18. Cai ZY, Yan Y, Sun SQ, et al. Minocycline attenuates cognitive impairment and restrains oxidative stress in the hippocampus of rats with chronic cerebral hypoperfusion. Neurosci Bull. 2008;24(5):305-313.

19. Cai ZY, Yan Y, Chen R. Minocycline reduces astrocytic reactivation and neuroinflammation in the hippocampus of a vascular cognitive impairment rat model. Neurosci Bull. 2010;26(1):28-36.

20. Zheng P, Zhang J, Liu H, Xu X, Zhang X. Angelica injection reduces cognitive impairment during chronic cerebral hypoperfusion through brain-derived neurotrophic factor and nerve growth factor. Curr Neurovasc Res. 2008;5(1):13-20. 
21. Kantor O, Schmitz C, Feiser J, et al. Moderate loss of cerebellar Purkinje cells after chronic bilateral common carotid artery occlusion in rats. Acta Neuropathol. 2007;113(5):549-558.

22. NIH committee recommends against revising the Guide. The Physiologist. 2007;50(1):23.

23. Wenk GL. Assessment of spatial memory using the radial arm maze and Morris water maze. Curr Protoc Neurosci. 2004; Chapter 8:Unit 8.5A.

24. Kohman RA, Bhattacharya TK, Kilby C, Bucko P, Rhodes JS. Effects of minocycline on spatial learning, hippocampal neurogenesis and microglia in aged and adult mice. Behav Brain Res. 2013;242:17-24.

25. Choi Y, Kim HS, Shin KY, et al. Minocycline attenuates neuronal cell death and improves cognitive impairment in Alzheimer's disease models. Neuropsychopharmacology. 2007;32(11):2393-2404.

26. Mattei D, Djodari-Irani A, Hadar R, et al. Minocycline rescues decrease in neurogenesis, increase in microglia cytokines and deficits in sensorimotor gating in an animal model of schizophrenia. Brain Behav Immun. 2014;38:175-184.

27. Xu L, Fagan SC, Waller JL, et al. Low dose intravenous minocycline is neuroprotective after middle cerebral artery occlusion-reperfusion in rats. BMC Neurol. 2004;4:7.

28. Yáñez M, Matías-Guiu J, Arranz-Tagarro JA, et al. The neuroprotection exerted by memantine, minocycline and lithium, against neurotoxicity of CSF from patients with amyotrophic lateral sclerosis, is antagonized by riluzole. Neurodegener Dis. 2014;13(2-3):171-179.

29. Ferretti MT, Allard S, Partridge V, Ducatenzeiler A, Cuello AC. Minocycline corrects early, pre-plaque neuroinflammation and inhibits BACE-1 in a transgenic model of Alzheimer's disease-like amyloid pathology. J Neuroinflammation. 2012;9:62.

30. Kumar A, Chaudhary T, Mishra J. Minocycline modulates neuroprotective effect of hesperidin against quinolinic acid induced Huntington's disease like symptoms in rats: behavioral, biochemical, cellular and histological evidences. Eur J Pharmacol. 2013;720(1-3):16-28.

31. Kalonia H, Mishra J, Kumar A. Targeting neuro-inflammatory cytokines and oxidative stress by minocycline attenuates quinolinic-acid-induced Huntington's disease-like symptoms in rats. Neurotox Res. 2012; 22(4):310-320.

32. Zhu F, Zheng Y, Ding YQ, et al. Minocycline and risperidone prevent microglia activation and rescue behavioral deficits induced by neonatal intrahippocampal injection of lipopolysaccharide in rats. PLoS One. 2014;9(4):e93966.

33. Abraham J, Fox PD, Condello C, Bartolini A, Koh S. Minocycline attenuates microglia activation and blocks the long-term epileptogenic effects of early-life seizures. Neurobiol Dis. 2012;46(2):425-430.

34. Soliman GM, Choi AO, Maysinger D, Winnik FM. Minocycline block copolymer micelles and their anti-inflammatory effects on microglia. Macromol Biosci. 2010;10(3):278-288.

35. Keller AF, Gravel M, Kriz J. Treatment with minocycline after disease onset alters astrocyte reactivity and increases microgliosis in SOD1 mutant mice. Exp Neurol. 2011;228(1):69-79.

36. Hinwood M, Tynan RJ, Charnley JL, Beynon SB, Day TA, Walker FR Chronic stress induced remodeling of the prefrontal cortex: structural re-organization of microglia and the inhibitory effect of minocycline. Cereb Cortex. 2013;23(8):1784-1797.

37. Chen SD, Yin JH, Hwang CS, Tang CM, Yang DI. Anti-apoptotic and anti-oxidative mechanisms of minocycline against sphingomyelinase/ ceramide neurotoxicity: implication in Alzheimer's disease and cerebral ischemia. Free Radic Res. 2012;46(8):940-950.

38. Liu X, Su H, Chu TH, Guo A, Wu W. Minocycline inhibited the proapoptotic effect of microglia on neural progenitor cells and protected their neuronal differentiation in vitro. Neurosci Lett. 2013;542: 30-36.

39. Kim J, Kwon JT, Kim HS, Josselyn SA, Han JH. Memory recall and modifications by activating neurons with elevated CREB. Nat Neurosci. 2014;17(1):65-72.

40. Scott Bitner R. Cyclic AMP response element-binding protein (CREB) phosphorylation: a mechanistic marker in the development of memory enhancing Alzheimer's disease therapeutics. Biochem Pharmacol. 2012; 83(6):705-714.
41. Suzuki A, Fukushima H, Mukawa T, et al. Upregulation of CREBmediated transcription enhances both short- and long-term memory. J Neurosci. 2011;31(24):8786-8802.

42. Wang B, Zhao J, Yu M, et al. Disturbance of Intracellular calcium homeostasis and CaMKII/CREB signaling is associated with learning and memory impairments induced by chronic aluminum exposure. Neurotox Res. 2014;26(1):52-63.

43. Nam SM, Choi JH, Yoo DY, et al. Effects of curcumin (Curcuma longa) on learning and spatial memory as well as cell proliferation and neuroblast differentiation in adult and aged mice by upregulating brainderived neurotrophic factor and CREB signaling. J Med Food. 2014; 17(6):641-649.

44. Kida S, Serita T. Functional roles of CREB as a positive regulator in the formation and enhancement of memory. Brain Res Bull. 2014;105: $17-24$.

45. Kim J, Kwon JT, Kim HS, Han JH. CREB and neuronal selection for memory trace. Front Neural Circuits. 2013;7:44.

46. Agerman K, Hjerling-Leffler J, Blanchard MP, et al. BDNF gene replacement reveals multiple mechanisms for establishing neurotrophin specificity during sensory nervous system development. Development. 2003;130(8):1479-1491.

47. Yu H, Zhang ZJ, Shi YM, et al. [Cognitive function, serum BDNF levels and BDNF gene Val66Met polymorphism in amnestic mild cognitive impairment]. Zhong Nan Da Xue Xue Bao Yi Xue Ban. 2008; 33(4):321-325. Chinese.

48. Kiprianova I, Sandkühler J, Schwab S, Hoyer S, Spranger M. Brainderived neurotrophic factor improves long-term potentiation and cognitive functions after transient forebrain ischemia in the rat. Exp Neurol. 1999;159(2):511-519.

49. Hellweg R, Jockers-Scherübl M. Neurotrophic factors in memory disorders. Life Sci. 1994;55(25-26):2165-2169.

50. Nacmias B, Piccini C, Bagnoli S, et al. Brain-derived neurotrophic factor, apolipoprotein $\mathrm{E}$ genetic variants and cognitive performance in Alzheimer's disease. Neurosci Lett. 2004;367(3):379-383.

51. Fossati P, Radtchenko A, Boyer P. Neuroplasticity: from MRI to depressive symptoms. Eur Neuropsychopharmacol. 2004;14 Suppl 5: S503-S510.

52. Duman RS. Pathophysiology of depression: the concept of synaptic plasticity. Eur Psychiatry. 2002;17 Suppl 3:306-310.

53. Alonso M, Medina JH, Pozzo-Miller L. ERK1/2 activation is necessary for BDNF to increase dendritic spine density in hippocampal CA1 pyramidal neurons. Learn Mem. 2004;11(2):172-178.

54. Pizarro JM, Lumley LA, Medina W, et al. Acute social defeat reduces neurotrophin expression in brain cortical and subcortical areas in mice. Brain Res. 2004;1025(1-2):10-20.

55. Berry A, Greco A, Giorgio M, et al. Deletion of the lifespan determinant p66(Shc) improves performance in a spatial memory task, decreases levels of oxidative stress markers in the hippocampus and increases levels of the neurotrophin BDNF in adult mice. Exp Gerontol. 2008;43(3):200-208.

56. Jain V, Baitharu I, Prasad D, Ilavazhagan G. Enriched environment prevents hypobaric hypoxia induced memory impairment and neurodegeneration: role of BDNF/PI3K/GSK3 $\beta$ pathway coupled with $\mathrm{CREB}$ activation. PLoS One. 2013;8(5):e62235.

57. Hattiangady B, Rao MS, Shetty GA, Shetty AK. Brain-derived neurotrophic factor, phosphorylated cyclic AMP response element binding protein and neuropeptide $\mathrm{Y}$ decline as early as middle age in the dentate gyrus and CA1 and CA3 subfields of the hippocampus. Exp Neurol.2005; 195(2):353-371.

58. Faverjon S, Silveira DC, Fu DD, et al. Beneficial effects of enriched environment following status epilepticus in immature rats. Neurology. 2002;59(9):1356-1364.

59. Chen DY, Bambah-Mukku D, Pollonini G, Alberini CM. Glucocorticoid receptors recruit the CaMKII $\alpha$-BDNF-CREB pathways to mediate memory consolidation. Nat Neurosci. 2012;15(12):1707-1714. 


\section{Publish your work in this journal}

Neuropsychiatric Disease and Treatment is an international, peerreviewed journal of clinical therapeutics and pharmacology focusing on concise rapid reporting of clinical or pre-clinical studies on a range of neuropsychiatric and neurological disorders. This journal is indexed on PubMed Central, the 'PsycINFO' database and CAS, and is the official journal of The International Neuropsychiatric Association (INA). The manuscript management system is completely online and includes a very quick and fair peer-review system, which is all easy to use. Visit http://www.dovepress.com/testimonials.php to read real quotes from published authors.

\footnotetext{
Submit your manuscript here: http://www.dovepress.com/neuropsychiatric-disease-and-treatment-journal
} 